\title{
Effectiveness of Compatibilizer on Mechanical Properties of Recycled PET Blends with PE, PP, and PS
}

\author{
Nobuyuki Imamura1, Hiroki Sakamoto², Yuji Higuchi², Hiroyuki Yamamoto³, \\ Shinichi Kawasaki ${ }^{3}$, Kazushi Yamada ${ }^{3}$, Hiroyuki Nishimura ${ }^{3}$, Takahiro Nishino ${ }^{4}$ \\ ${ }^{1}$ Osaka Resin Industry Co., Osaka, Japan \\ ${ }^{2}$ Energy Technology Laboratories, Osaka Gas Co., Osaka, Japan \\ ${ }^{3}$ Advanced Fibro-Science, Kyoto Institute of Technology, Kyoto, Japan \\ ${ }^{4}$ Osaka Gas Chemicals Co., Konohana-ku, Osaka, Japan \\ Email: ${ }^{*}$ kazushi@kit.ac.jp
}

Received 23 April 2014; revised 25 May 2014; accepted 9 June 2014

Copyright (C) 2014 by authors and Scientific Research Publishing Inc.

This work is licensed under the Creative Commons Attribution International License (CC BY).

http://creativecommons.org/licenses/by/4.0/

c) (i) Open Access

\begin{abstract}
Recycled PET (R-PET) is well known to exhibit brittle behavior in the presence of notches and indicated the low heat distortion temperature. In addition, it is hard to prevent some impurities such as cap or label of the bottle that mixed into R-PET during the recycling process. In this paper, the effect of the amounts and kinds of compatibilizers on the morphological characteristics and mechanical performance of recycled poly(ethylene terephthalate) (R-PET) compounded with polyethylene (PE), polypropylene (PP), and polystyrene (PS) was investigated. From the results, with an increase in the glycidyl methacrylate modified PE (EGMA) additive contents, in the increment of the Izod impact strength of Composite-G was obtained. In addition, it was found that the miscibility of Composite-G was improved with increasing the amount of EGMA, which indicated from the result of SEM images.
\end{abstract}

\section{Keywords}

Component, Recycled PET, Recycled Polypropylene, Recycled Polyethylene, Compatibilizer

\section{Introduction}

In recent years, poly(ethylene terephthalate) (PET) is widely used for engineering plastic such as for making soft *Corresponding author.

How to cite this paper: Imamura, N., Sakamoto, H., Higuchi, Y., Yamamoto, H., Kawasaki, S., Yamada, K., Nishimura, H. and Nishino, T. (2014) Effectiveness of Compatibilizer on Mechanical Properties of Recycled PET Blends with PE, PP, and PS. Materials Sciences and Applications, 5, 548-555. http://dx.doi.org/10.4236/msa.2014.58057 
drink bottles. The reason is mainly attributed to its transparency, thermal stability, chemical resistance, and excellent barrier properties of PET. On the other hand, a cap of the bottles is made from polyethylene (PE) or polypropylene (PP) due to the flexibility and formability while a label produces from polystyrene (PS) film. In recycling process, it is important to remove the PP or PE caps and PS labels from waste PET bottles in order to get the high purity of recycled-PET (RPET) flake materials. However, some unnecessary polymers could be remained and be mixed in RPET materials. In addition, it is well known that the mechanical properties of the recycled materials would decrease due to the decomposition and degradation of polymer chains. Some investigations have been concerning the control of degradation in polymers during recycling by creating blends and/or introducing additives. Several studies have been reported in the literatures on the usage of a third polymer component for compatibilized PET and polyolefin blends in order to improve the mechanical properties, thermal stability, and toughness of the blends. The improvements of toughness for such blends have been reported with the compounding of ethylene-glycidyl methacrylate copolymer (EGMA) in RPET [1]-[4]. On the other hand, the fillers such as talc or $\mathrm{CaCO}_{3}$ were used in order to improve the thermal resistance and flame retardancy of RPET/RPP blends [5]-[9]. Inoya et al. [10] [11] has reported on hydrogenated block-copolymer of SEBS for improving the compatibility of R-PET with PP blends. Many investigations had been performed on polymer blends involving RPET bottles from post-consumer waste PET bottles with discarding caps and labels. Therefore, it is important to focus on the effect of bottle caps and labels on the homogeneity and mechanical performance of the blends from using the post-consumer waste PET bottles. It can be noted that impact strength of RPET blends can be obtained by compatibilization, which is usually attained by functionalization of the rubber phase.

The aim of this study is to produce recycled materials with high static mechanical performances and impact strengths using the post-consumer PET bottles by blending waste PET with waste bottle caps, labels and compatibilizers. The effect of the amounts and types of compatibilizerson properties of recycled RPET blends was investigated. The structure and properties of these recycled PET based blends prepared by reactive compounding were carried out on the basis of mechanical testing and morphological characteristics.

\section{Experimental}

\subsection{Materials}

Materials used in this study were recycled poly(ethylene terephthalate) (RPET) supplied by Osaka Prefecture in Japan. Figure 1 shows a photograph of the collected a post-consumer waste PET bottle including a bottle, a cap, and a label, which were PET, PE or PP, and PS, respectively. Glycidyl methacrylate modified PE copolymer (EGMA) and $\alpha$-olefin modified linear low-density-polyethylene copolymer (LLE) as shown in Figure 2 were used as compatibilizers for blending RPET with PE, PP and PS. The chemical structures of these compatibilizers are compatible with RPET due to the presence of epoxy or hydroxyl functional groups that could react and/or interact with the carboxyl end groups of PET. Therefore, it can either work as a compatibilizer or an impact modifier for these compositions.

\subsection{Sample Preparation}

The sample designation and the composition of the composites are shown in Table 1 and Table 2, respectively. The RPET samples were compatibilized with EGMA, LLE and hybrid of EGMA and LLE are referred as Composite-G, Composite-L and Composite-GL, respectively. The extruder barrel temperature was set between $250^{\circ} \mathrm{C}$ and $260^{\circ} \mathrm{C}$, at screw speeds of 100 and $200 \mathrm{rpm}$. RPET composite pellets as shown in Figure 3 were pelletized from the strands in this condition. The pellets were dried with a dehumidifying drier at $80^{\circ} \mathrm{C}$ for $5 \mathrm{~h}$ before injection-molded (UM50, Po Yuen Co., Ltd., Hong Kong, China) into dumbbell specimens at barrel temperatures of $260^{\circ} \mathrm{C}-280^{\circ} \mathrm{C}$ at an injection speed of $100 \mathrm{~mm} / \mathrm{s}$.

\subsection{Mechanical Tests}

Tensile tests were performed by using an Instron 4206 universal testing machine according to JIS K7113. The gauge length of the specimens was $115 \mathrm{~mm}$ and the tests were conducted at an extension rate of $50 \mathrm{~mm} / \mathrm{min}$.

Izod impact strength was determined for all specimens that were notched at $2 \mathrm{~mm}$-depth. The impact specimens were obtained from the parallel regions of the dumbbell specimens. The tests were conducted by using a 


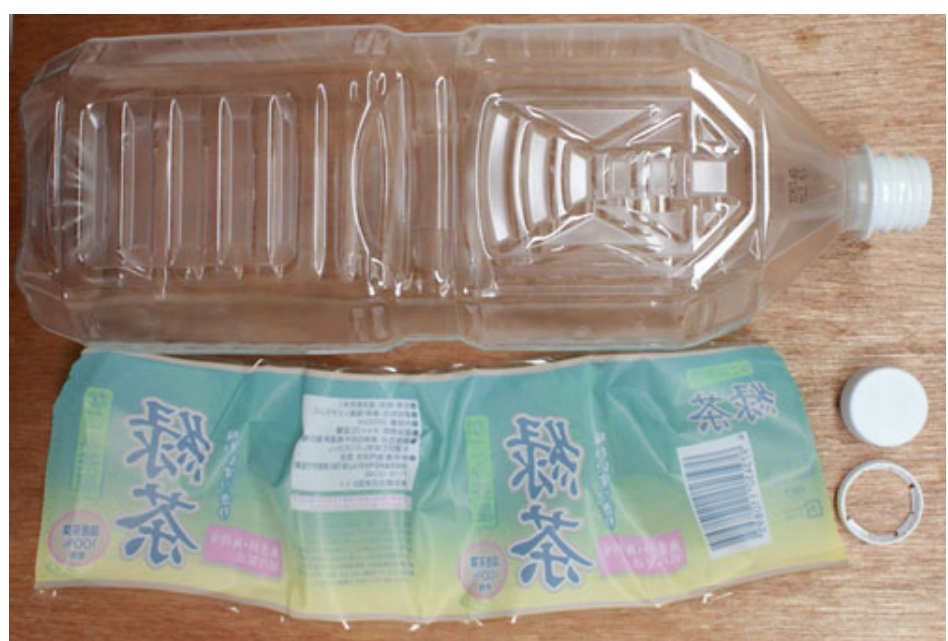

Figure 1. Photograph of waste PET bottle, PE or PP cap, and PS label.<smiles>CCCCCC(C)(C)CC(C)(C)C(=O)OCC1CO1</smiles><smiles>[1H]C(C)(CC)CCC(C)(C)C=C</smiles>

Figure 2. Chemical structure of (a) EGMA (Poly(ethylene-co-glycidyl methacrylate) copolymer) and (b) LLE ( $\alpha$-olefin modified linear low-density-polyethylene copolymer).

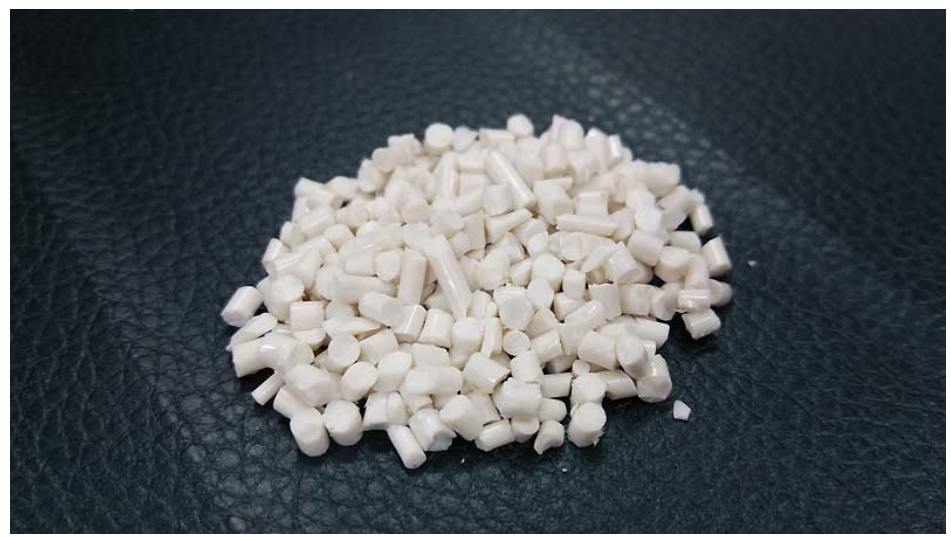

Figure 3. Photograph of RPET pellets.

Table 1. Component ratio of RPET pellet.

\begin{tabular}{ccccc}
\hline Polymer & PET & PE & PP & PS \\
\hline wt $\%$ & 90 & 4 & 3 & 3 \\
\hline
\end{tabular}


Table 2. Component ratio of RPET composites blended with various compatibilizers (unit: phr).

\begin{tabular}{cccc}
\hline & RPET & EGMA & LLE \\
RPET & 100 & & \\
Composite-G3 & 100 & 3 & \\
Composite-G10 & 100 & 10 & \\
Composite-G15 & 100 & 15 & 3 \\
Composite-L3 & 100 & & 10 \\
Composite-L10 & 100 & & 15 \\
Composite-L15 & 100 & 1 & 7 \\
Composite-GL3 & 100 & 3 & 10 \\
Composite-GL10 & 100 & 5 & \\
Composite-GL15 & 100 & & \\
\hline
\end{tabular}

Toyo Seiki Izod impact tester in accordance to ASTM D256 with a pendulum of $5.50 \mathrm{~J}$.

\subsection{Scanning Electron Microscope}

Morphology of the blends was characterized from tensile fractured specimens by using a scanning electron microscope (JEOL, JSM-6010-LA). Gold coating was sputtered onto the specimens for the purpose of enhancing conductivity.

\section{Results and Discussion}

\subsection{Mechanical Properties for Composites}

Figure 4 shows the result of the tensile modulus of RPET and RPET composites with various compatibilizers. The tensile modulus of these composites decreased with increasing the amount of compatibilizers for RPET, which was due to the flexibility of the EGMA and LLE copolymers. It can be noted that the tensile modulus of Composite-L slightly increased when compounded with LLE at 15 phr. It might be attributed to higher molecular entanglement between RPET with LLE, which improved the tensile modulus of the composite-L at 15 phr of LLE.

Figure 5 shows the tensile strength of RET and various RPET composites compounded with compatibilizers. The tensile strength of Composite-G, L, and GL was lower than neat-RPET and the tensile strengths of RPET composites gradually decreased with increasing the contents of compatibilizers for RPET. It was due to flexibility of the composites G, L and GL and poor stress transferring between the composites molecule. Therefore, the tensile strength of the composites decreased when adding higher amount of compatibilizers.

Figure 6 shows the results of elongation at break for these RPET composites. As shown in the figure, the elongation at break of each composite increased with increasing the amount of compatibilizers for RPET. It was interesting to note that the elongation at break of all composites was higher than neat-RPET. These were corresponding to the improving of flexibility and elongated of the RPET composites when incorporated with compatibilizers. In addition, at 3 phr of compatibilizers contents, the elongation at break of composite-G showed the highest value as compared with all composites. On the other hand, of the elongation at break of Composite-GL indicated the highest value at 15 phr content. Figure 7 shows the typical photographs of dumbbell specimens of (a) RPET and (b) Composite-GL15 after tensile testing. It can be considered that the EGMA and the LLE compatibilizers played an important role in order to improve the tensile properties for RPET composites blended with PE, PP, and PS.

Figure 8 shows the result of Izod impact strength of RPET and various composites. The Izod impact strength increased with increasing the amount of compatibilizer for RPET and higher than neat RPET. It can be seen that the Izod impact strength of Composite-G drastically increased with increasing the amount of compatibilizer contents as compared with Composite-L and Composite-GL. On the other hand, the Izod impact strength of Composite-L slightly increased with increasing the amount of LLE content. 


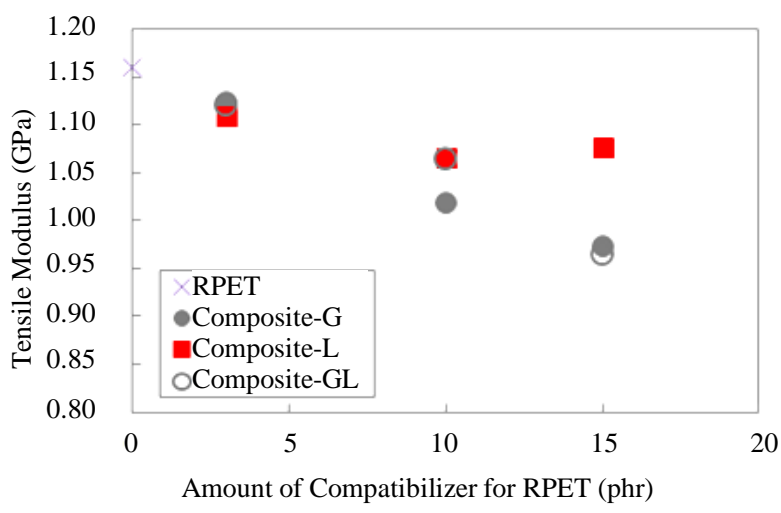

Figure 4. Tensile modulus of RPET and various composites.

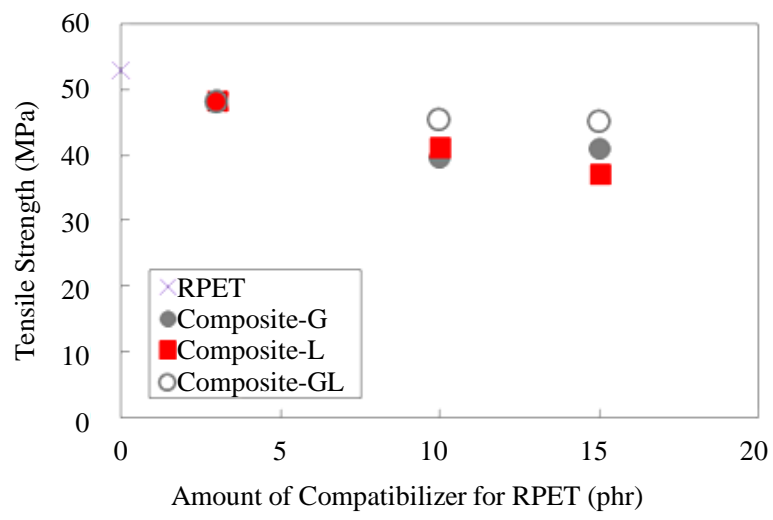

Figure 5. Tensile strength of RPET and various composites.

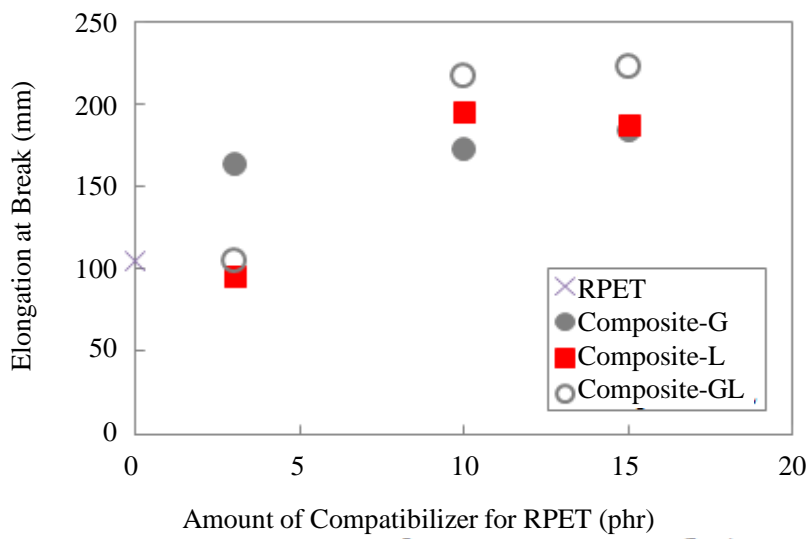

Figure 6. Elongation at break of RPET and various composites.

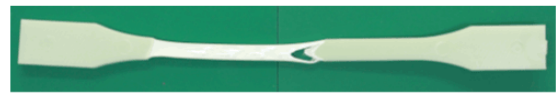

(a)

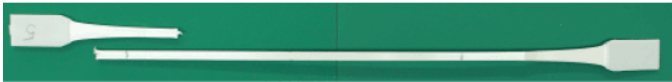

(b)

Figure 7. Photographs of dumbbell specimens after tensile test, (a) RPET and (b) Composite-GL (15 phr). 


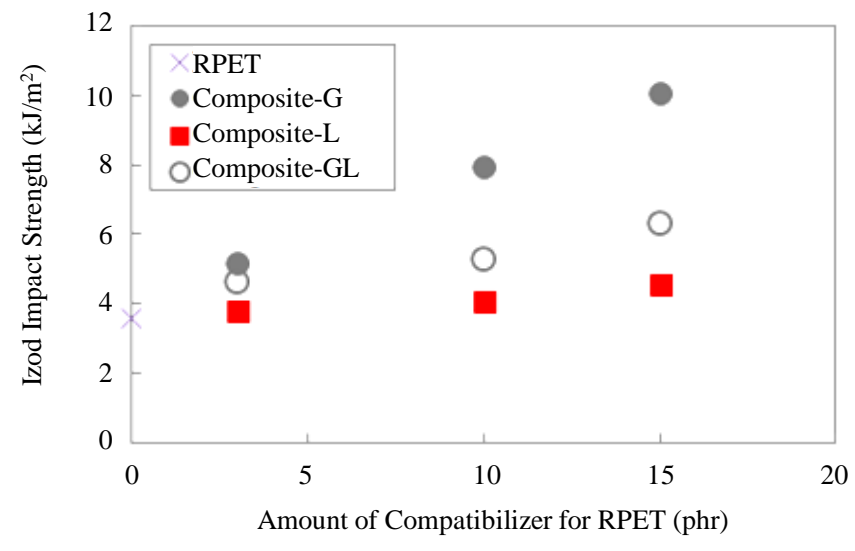

Figure 8. Izod impact strength of RPET and various composites.

In the RPET/EGMA blends, the EGMA phase reacted with the hydroxyl groups from RPET to form a copolymer in situ, which was responsible for the uniform dispersion of EGMA in RPET. Similarly, the carboxyl and hydroxyl groups in RPET reacted with the epoxy group from EGMA in the melt state thus increased the molecular weights of RPET. Therefore, we suggest that the molecular weight of the blends increased because of the strong affinity between the RPET and EGMA phases. On the other hand, in the RPET/LLE blends, the LLE has double bond as end group of linear olefin molecules, which has no functional group such as epoxy groups and hydroxyl groups. Accordingly, it is considered that the LLE indicated the good miscibility with PE, PP, and PS molecules as compared with PET molecules. In addition, the double bond of LLE has weakly-reaction with carboxyl- and hydroxyl-groups of PET molecules. As a result, the LLE compatibilizer is not considered as effective for the tensile properties in Composite-L as compared with the EGMA compatibilizer for RPET. Furthermore, it can be noted that the crystallinity and the density of the blends indicated a tendency of decreasing with increasing the amount of compatibilizer as described in Ref.1. Hence, we consider that the tensile properties were depended on the crystallinity and the density of the blends. From the literature, the homogeneity of the RPET blends was affected by the miscibility and the reaction between RPET and compatibilizers. From this interesting result, one considers is the mechanical performance of the RPET blends with PE, PP, and PS could be increased by the variation of compatibilizers.

\subsection{Morphology Observation}

Figures 9-11 show SEM micrographs from the tensile fractured surfaces of blends prepared at various compatibilizer contents. In general, a brittle fracture surface could be expected from the unmodified RPET, similar with virgin PET, because it is known to be very notch-sensitive and readily-crystallized condition. However, as shown in Figure 9, the RPET exhibited dispersed phase of other polymers such as PE, PP, and PS. PET is a polar polymer with include carboxyl and hydroxyl groups in the molecules, which would not show the miscibility with non-polar polymers such as PE, PP, and PS. Therefore, it is considered that the small dispersed phase particle structure corresponded to PE, PP, or PS was observed on the fracture surface of RPET and RPET composites. On the other hand, the SEM micrographs of tensile fractured surfaces of blends with compatibilizer are shown in Figure 10 and Figure 11. As shown in these SEM images, the fractured surface of Composite-G was changed with increasing the amount of EGMA content, i.e. the roughness of the fracture surface becomes small due to the compatibilizing effect of EGMA for PET and PE, PP, PS molecules. It is considered that the decrease of the roughness was attributed to the improvement of phase-separation structure between RPET and dispersed phase of other polymers. On the other hand, the morphology of the surface fracture for Composite-L did not indicate a change with increasing the amount of LLE contents. It was considered that this could support the results of tensile testing for Composite-L. The fracture surface of Composite-GL exhibited the average of surface roughness between Composite-G and Composite-L. This result was well corresponding to the results of Izod impact strength. In these results, the LLE compatibilizer is not so effective for improving the impact strength of RPET blends. However, it was found that the EGMA compatibilizer plays an important role to improve the miscibility, which is not only PET and PP, but also improved miscibility between PET and PE, PS molecules. As a 

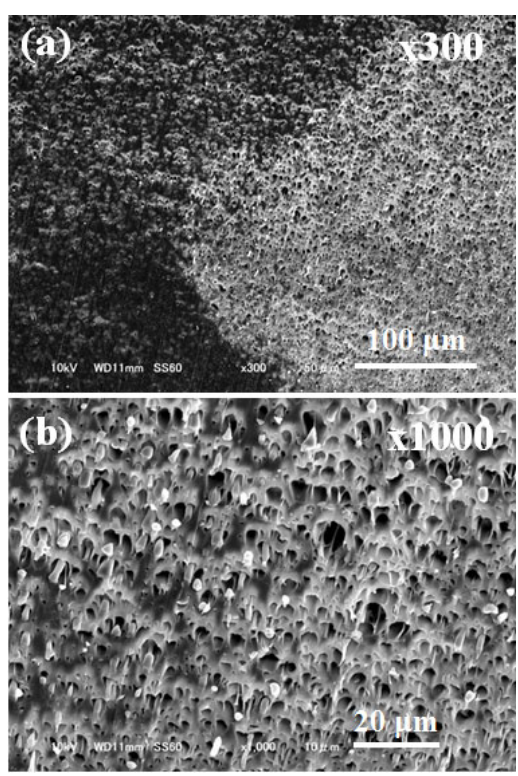

Figure 9. SEM images of fracture surface for RPET specimens after tensile test.

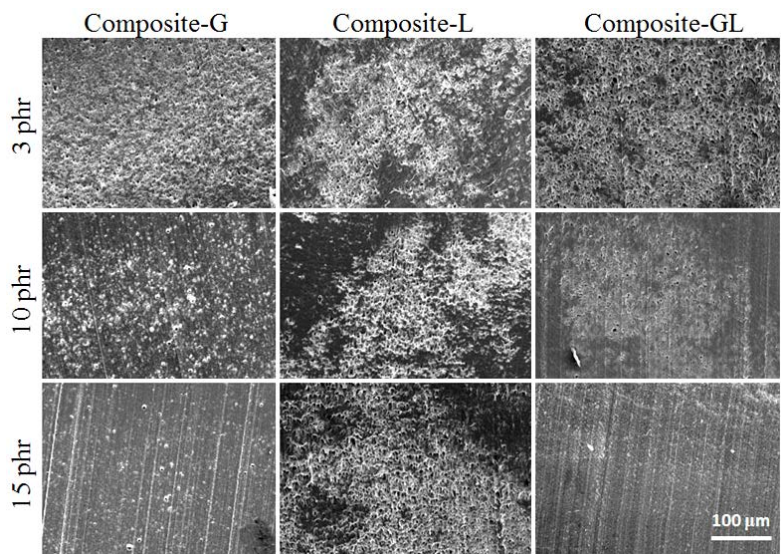

Figure 10. SEM images of fracture surface for various composite specimens after tensile test (Magnification is $\times 300$ ).

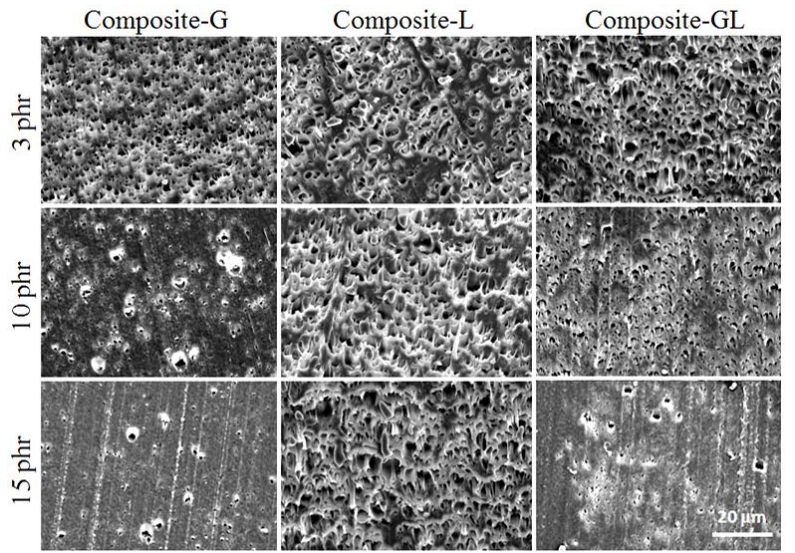

Figure 11. SEM images of fracture surface for various composite specimens after tensile test (Magnification is $\times 1000$ ). 
result, we demonstrated the possibility of a novel PET bottle recycling method, that is, the recycling of a whole waste PET bottle with PE-PP cap and PS label by using the EGMA compatibilizer.

\section{Conclusion}

In this investigation, we tried to elucidate the effect of compatibilizer for recycled PET bottle with waste bottle caps and labels, i.e. the composites consist of PET, PE, PP, PS, and compatibilizer blends. This research produces recycled materials with high static mechanical performances and impact strengths by blending waste PET with waste bottle caps, labels, and compatibilizers. As a result, it was found that the EGMA compatibilizer plays an important role in improving the miscibility with not only PET and PP, but also PET and PE, PS molecules. Nevertheless, the LLE compatibilizer does not work so well for the impact strength of RPET blends. In this paper, we demonstrated the possibility of a novel PET bottle recycling method, that is, the recycling of a whole PET bottle with PE-PP cap and PS label by using EGMA compatibilizer. We hope this research result make a contribution for the reduction of environmental burdens and the PET bottle recycling.

\section{References}

[1] Kunimune, N., Yamada, K., Leong, Y.W., Thumsorn, S. and Hamada, H. (2011) Influence of the Reactive Processing of Recycled Poly(ethylene terephthalate)/Poly(ethylene-co-glycidyl methacrylate) Blends. Journal of Applied Polymer Science, 120, 50-55. http://dx.doi.org/10.1002/app.32836

[2] Pietrasanta, Y., Robin, J.-J., Torres, N. and Boutevin, B. (1999) Reactive Compatibilization of HDPE/PET Blends by Glycidyl Methacrylate Functionalized Polyolefins. Macromolecular Chemistry and Physics, 200, 142-149.

[3] Pracella, M., Pazzagli, F. and Galeski, A. (2002) Reactive Compatibilization and Properties of Recycled Poly(Ethylene Terephthalate)/Polyethylene Blends. Polymer Bulletin, 48, 67-74. http://dx.doi.org/10.1007/s00289-002-0001-7

[4] Papadopoulou, C.P. and Kalfoglou, N.K. (2000) Comparison of Compatibilizer Effectiveness for PET/PP Blends: Their Mechanical, Thermal and Morphology Characterization. Polymer, 41, 2543-2555. http://dx.doi.org/10.1016/S0032-3861(99)00442-5

[5] Thumsorn, S., Yamada, K., Leong, Y.W. and Hamada, H. (2011) Development of Cockleshell-Derived CaCO 3 for Flame Retardancy of Recycled PET/Recycled PP Blend. Materials Sciences and Applications, 2, 59-69. http://dx.doi.org/10.4236/msa.2011.22009

[6] Thumsorn, S., Yamada, K., Leong, Y.W. and Hamada, H. (2012) Effect of Pellet Size and Compatibilization on Thermal Decomposition Kinetic of Recycled Polyethylene Terephthalate/Recycled Polypropylene Blend. Journal of Applied Polymer Science, 124, 1605-1613. http://dx.doi.org/10.1002/app.35166

[7] Ogasahara, M., Shidou, M., Nagata, S., Yamada, K., Leong, Y.W. and Hamada, H. (2012) Effectiveness of High Frequency Heating on Drying and Intrinsic Viscosity Enhancement of Recycled Poly(Ethylene Terephthalate). Journal of Applied Polymer Science, 126, E166-E171. http://dx.doi.org/10.1002/app.34368

[8] Thumsorn, S., Yamada, K., Leong, Y.W. and Hamada, H. (2013) Thermal Decomposition Kinetic and Flame Retardancy of $\mathrm{CaCO}_{3}$ Filled Recycled Polyethylene Terephthalate/Recycled Polypropylene Blend. Journal of Applied Polymer Science, 127, 1245-1256. http://dx.doi.org/10.1002/app.37673

[9] Yamada, K. and Thumsorn, S. (2013) Effectiveness of Talc Filler on Thermal Resistance of Recycled PET Blends. Advances in Materials Physics and Chemistry, 3, 327-331. http://dx.doi.org/10.4236/ampc.2013.38045

[10] Inoya, H., Leong, Y.W., Klinklai, W., Thumsorn, S., Makata, Y. and Hamada, H. (2012) Compatibilization of Recycled Poly(Ethylene Terephthalate) and Polypropylene Blends: Effect of Polypropylene Molecular Weight on Homogeneity and Compatibility. Journal of Applied Polymer Science, 124, 3947-3955. http://dx.doi.org/10.1002/app.34405

[11] Inoya, H., Leong, Y.W., Klinklai, W., Takai, Y. and Hamada, H. (2012) Compatibilization of Recycled Poly(Ethylene Terephthalate) and Polypropylene Blends: Effect of Compatibilization on Blend Toughness, Dispersion of Minor Phase, and Thermal Stability. Journal of Applied Polymer Science, 124, 5260-5269.

http://dx.doi.org/10.1002/app.34385 
Scientific Research Publishing (SCIRP) is one of the largest Open Access journal publishers. It is currently publishing more than 200 open access, online, peer-reviewed journals covering a wide range of academic disciplines. SCIRP serves the worldwide academic communities and contributes to the progress and application of science with its publication.

Other selected journals from SCIRP are listed as below. Submit your manuscript to us via either submit@scirp.org or Online Submission Portal.
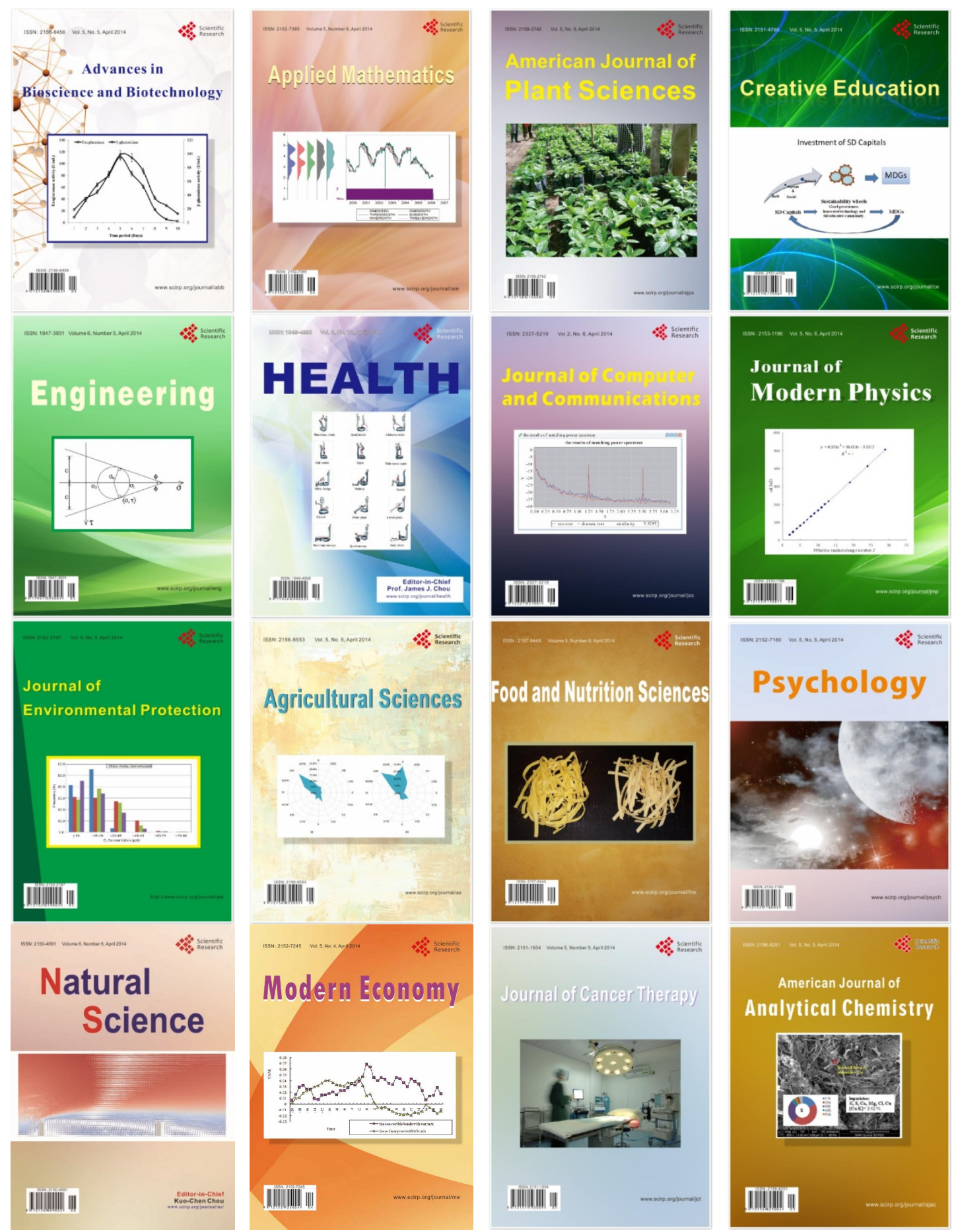